VOL. $4(1971), 259-262$.

\title{
Convergence criteria for Fourier series
}

\section{Venu Gopal Rao}

The following convergence criterion of Fourier series is due to M. Izumi, S. Izumi and the author:

THEOREM. Let $\Delta \geq 1$. If

(i) $\int_{0}^{t} \phi(u) d u=o(t)$, and

(ii) $\int_{t^{1 / \Delta}}^{\delta}\left|a\left(u^{-a} \phi(u)\right)\right| \leq A t^{-a}$ as $t \rightarrow 0$

for an $a, 0<a<1$ and for $a \delta, 0<\delta<\pi$, then the Fourier series of $\phi(t)$ is convergent at the origin.

The object of this paper is to generalize the above theorem in the Hardy-Lit+lewood direction.

1 .

Let $\phi(t)$ be an even periodic function which is integrable $L$ and let

$$
\phi(t) \sim \sum_{n=1}^{\infty} a_{n} \cos n t .
$$

Sunouch i [4] generalized the Young-Pollard [3]-convergence criterion as follows:

THEOREM A. The Fourier series of $\phi(t)$ converges at the point $t=0$ to the value zero, provided that there is a $\Delta \geq 1$ such that

Received 13 November 1970. The author wishes to express his thanks to Mrs Masako Izumi and Dr Shin-ichi Izumi for their interest during the preparation of this paper. 


$$
\int_{0}^{t} \phi(u) d u=o\left(t^{\Delta}\right)
$$

and

$$
\int_{0}^{t}\left|d\left(u^{\Delta} \phi(u)\right)\right|=o(t), 0 \leq t \leq n .
$$

Recently we [2] proved the following theorem:

THEOREM B. Let $\Delta \geq 1$. If the condition (1.1) holds and

$$
\int_{t^{I / \Delta}}^{\delta} \mid d\left(u^{\left.-a_{\phi}(u)\right)} \mid=O\left(t^{-a}\right) \text { as } t \rightarrow 0\right.
$$

for an $a, 0<a<1$ and for $a \delta, 0<\delta<\pi$, then the Fourier series of $\phi$ is convergent at the origin.

Hardy and Littlewood [1] generalized the condition (1.1) for the case $\Delta=1$ in the form

$$
\phi_{B}(t)=o\left(t^{\gamma}\right) \text {, as } t \rightarrow 0
$$

for any $B>0$, where $\phi_{\beta}(t)$ is the $\beta$-th integral of $\phi(t)$.

Corresponding to this result we prove the following theorem, which generalizes Theorem B in the Hardy-Lit+lewood direction.

THEOREM. Let $\Delta=\gamma / \beta \geq 1$ and $1>\beta>0$. If

$$
\phi_{B}(t)=o\left(t^{\gamma}\right)
$$

where $\phi_{B}(t)$ is the $B$-th integral of $\phi(t)$, and further if

$$
\int_{t^{1 / \Delta}}^{\delta}\left|a\left(u^{-n_{\phi}}(u)\right)\right|=o\left(t^{-n}\right), \quad 1>n>0,
$$

and $\Delta>1$, then the Fourier series of $\phi(t)$ converges at $t=0$.

2.

Proof of the theorem. To prove our theorem we need to show that 


$$
\int_{0}^{\delta} \phi(t) \frac{\sin n t}{t} d t=o(1) \text { as } n+\infty
$$

Putting $n^{-1 / \Delta}=\alpha$, we have

$$
\begin{aligned}
\int_{0}^{\delta} \phi(t) \frac{\operatorname{sinn} t}{t} d t & =\int_{0}^{\alpha} \phi(t) \frac{\operatorname{sinn} t}{t} d t+\int_{\alpha}^{\delta} \phi(t) \frac{\operatorname{sinn} t}{t} d t \\
& =I+J,
\end{aligned}
$$

say.

$$
\begin{gathered}
\text { Putting } \theta(t)=t^{-\eta} \phi(t) \text {, then } \theta(t)=o\left(t^{-n \Delta}\right) \text { by (1.5). Since } \\
\theta(t)=\int_{t}^{\delta} \frac{\operatorname{sinnu}}{u^{1-n}} d u=o\left(\frac{1}{n t^{1-n}}\right) \text { as } t \rightarrow 0,
\end{gathered}
$$

we get

$$
\begin{aligned}
& J=[\theta(t) \theta(t)]_{\alpha}^{\delta}-\int_{\alpha}^{\delta} \theta(t) d \theta(t)
\end{aligned}
$$

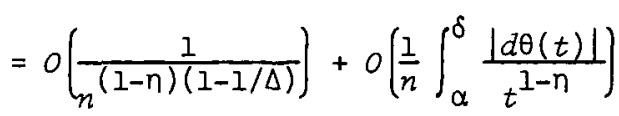

$$
\begin{aligned}
& =o\left(\frac{1}{n(1-\eta)(1-1 / \Delta)}\right)=o(1) \text { as } n \rightarrow \infty \text {. }
\end{aligned}
$$

We shall now estimate $I$.

Putting $\Phi(t)=\int_{0}^{t} \phi(u) d u$ and integrating by parts we have

$$
\begin{aligned}
I & =\left[\Phi(t) \frac{\sin n t}{t}\right]_{0}^{\alpha}-\int_{0}^{\alpha} \Phi(t) \frac{n t \cos n t-\sin n t}{t^{2}} d t \\
& =I_{1}+I_{2}
\end{aligned}
$$

say. Since

$$
\Phi(t)=o\left(t^{1+\gamma-\beta}\right)=o(t)
$$

by $(1.4)$, we get

$$
I_{1}=o(1) \text {. }
$$


Finally

$$
\begin{aligned}
I_{2} & =\int_{0}^{\alpha} \frac{n t \cos n t-\sin n t}{t^{2}} d t \int_{0}^{t} \phi_{B}(t)(t-u)^{-\beta} d u \\
& =\int_{0}^{\alpha} \phi_{B}(u) d u \int_{u}^{\alpha} \frac{n t \cos n t-\sin n t}{t^{2}}(t-u)^{-\beta} d t
\end{aligned}
$$

where the inner integral becomes

$$
n^{1+\beta} \int_{n u}^{n \alpha} \frac{\tau \cos \tau-\sin \tau}{\tau^{2}}(\tau-n u)^{-\beta} d \tau=o\left(\frac{n^{\beta}}{u}\right) .
$$

Thus we get

$$
\begin{aligned}
I_{2} & =o\left(n^{\beta} \int_{0}^{\alpha} \frac{\phi_{\beta}(u)}{u} d u\right) \\
& =o\left(n^{\beta} \int_{0}^{\alpha} \frac{u^{\gamma}}{u} d u\right) \\
& =o(1) .
\end{aligned}
$$

This completes the proof of the theorem.

\section{References}

[1] G.H. Hardy and J.E. Littlewood, "Notes on the theory of series (VII): On Young's convergence criterion for Fourier series", Proc. London Math. Soc. (2) 28 (1928), 301-311.

[2] Masako Izumi, Shin-ichi Izumi and V.V. Gopal Rao, "On the convergence criteria of Fourier series", Proc. Japan Acad. (to appear).

[3] S. Pollard, "On the criteria for the convergence of a Fourier series", J. London Math. Soc. 2 (1927), 255-262.

[4] Gen-ichirô Sunouchi, "Notes on Fourier analysis. XLVI. A convergence criterion for Fourier series", Tôhoku Math. J. (2) 3 (1951), 216-219.

Institute of Advanced Studies, Australian National University, Canberra, ACT. 